\title{
Ridge-Valley Lines on Meshes via Implicit Surface Fitting
}

\author{
Yutaka Ohtake $^{1,2} \quad$ Alexander Belyaev $^{2} \quad$ Hans-Peter Seidel $^{2}$ \\ ${ }^{1}$ RIKEN $\quad{ }^{2}$ Max-Planck-Institut für Informatik
}

\begin{abstract}
We propose a simple and effective method for detecting view- and scale-independent ridge-valley lines defined via first- and secondorder curvature derivatives on shapes approximated by dense triangle meshes. A high-quality estimation of high-order surface derivatives is achieved by combining multi-level implicit surface fitting and finite difference approximations. We demonstrate that the ridges and valleys are geometrically and perceptually salient surface features and, therefore, can be potentially used for shape recognition, coding, and quality evaluation purposes.
\end{abstract}

Keywords: ridges, curvature extrema, implicit surface fitting.

\section{Introduction}

Ridge-valley lines, curves on a surface along which the surface bends sharply, are powerful shape descriptors. Robust extraction of ridge-valley structures is important for image analysis [Monga et al. 1992; López et al. 1998], quality control of free-form surfaces [Hosaka 1992], geomorphology [Little and Shi 2001], analysis and registration of anatomical structures [Grenader and Miller 1998; Pennec et al. 2000], and face recognition [Hallinan et al. 1999]. (See also references therein.) In geometric modeling, there has been considerable effort to develop robust methods for crease detection on dense triangle meshes [Watanabe and Belyaev 2001; Hubeli and Gross 2001; Page et al. 2002; Stylianou and Farin 2003] and pointsampled surfaces [Gumhold et al. 2001; Pauly et al. 2003].

In this paper, we deal with surface creases defined via extrema of the principal curvatures along their corresponding curvature lines. Our motivation to consider curvature extrema is based upon the following analogy with edges of grey-scale images.

A widely used definition for edges in image processing describes them as the sets of pixels where the magnitude of the gradient of the image intensity has a local maximum in the direction of the gradient. Consider a surface $\mathscr{S}$ and its Gauss map which associates with every point $\mathbf{p}$ of $\mathscr{S}$ the oriented normal vector $\mathbf{n}(\mathbf{p})$. The derivative $\nabla \mathbf{n}(\mathbf{p})$ (Jacobian matrix) of the Gauss map measures the variation of the normal vector near $\mathbf{p}$, i.e., how the surface bends near $\mathbf{p}$. Since the eigenvalues and eigenvectors of $\nabla \mathbf{n}(\mathbf{p})$ are the principal curvatures and principal directions of $\mathscr{S}$ at $\mathbf{p}$, respectively, the maximal variation the surface normal is achieved in the principal direction of the principal curvature maximal in absolute value. Thus it is natural to define surface creases as loci of points where the positive (negative) variation of the surface normal in the direction of its maximal change attains a local maximum (minimum). We have arrived at the following definition of surface creases: the loci of points where the largest in absolute value principal curvature takes a positive maximum (negative minimum) along its corresponding curvature line.

Permission to make digital or hard copies of part or all of this work for personal or classroom use is granted without fee provided that copies are not made or distributed for profit or direct commercial advantage and that copies show this notice on the first page or initial screen of a display along with the full citation. Copyrights for components of this work owned by others than ACM must be honored. Abstracting with credit is permitted. To copy otherwise, to republish, to post on servers, to redistribute to lists, or to use any component of this work in other works requires prior specific permission and/or a fee. Permissions may be requested from Publications Dept., ACM, Inc., 1515 Broadway, New York, NY 10036 USA, fax +1 (212) 869-0481, or permissions@acm.org.

(c) 2004 ACM 0730-0301/04/0800-0609 $\$ 5.00$
The extrema of the principal curvatures along their corresponding curvature lines can be described as surface points where osculating spheres have high-order contacts with the surface. See [Koenderink 1990; Porteous 1994; Belyaev et al. 1997]; [Hallinan et al. 1999, Chapter 6], and references therein for rigorous mathematical treatments revealing beautiful properties of these curvature features. However practical detection of curvature extrema is widely considered a difficult computational task since, it involves estimating of high-order surface derivatives.

Developing methods for reliable computation of curvature measures for discrete surfaces is currently a subject of intensive research. The two main approaches to curvature tensor estimation consist of using finite differences [Meyer et al. 2003; Cohen-Steiner and Morvan 2003] and polynomial fitting [Cazals and Pouget 2003; Goldfeather and Interrante 2004]. A robust estimation of first-order curvature derivatives may require a combination of both these approaches [Stylianou and Farin 2003]. In general, local methods show worse performance in estimating curvature derivatives than global ones. Indeed, moving from one mesh vertex to another changes discontinuously the local vertex neighborhood where a local curvature estimation scheme is used and results in jerky behavior of estimated curvatures and curvature derivatives.

The idea of using global smoothing methods for robust detection of curvature extrema on height data was considered in [Kent et al. 1996] where globally supported radial basis functions (RBFs) were used for smoothing. Unfortunately, a straightforward use of globally supported RBFs for interpolating and approximating large 3D data is computationally expensive, and sophisticated mathematical techniques are needed to accelerate the fitting procedure [Carr et al. 2001]. In our work, we employ compactly supported radial basis functions (CS-RBFs) for global smoothing purposes. Specifically, we use a slight modification of the method developed in [Ohtake et al. 2003b] which is fast, not difficult to implement, and can be used either for approximation or interpolation.

Given a triangle mesh, we approximate it by a CS-RBF surface and project the mesh vertices onto the surface. The curvature tensor and curvature derivatives at a mesh vertex are estimated by those at the corresponding surface point. Finally we detect curvature maxima and minima on mesh edges and trace ridges and valleys.

We believe that in this paper, for the first time, a reliable detection of ridge-valley structures defined via first- and secondorder curvature derivatives is achieved on surfaces approximated by dense triangle meshes generated from typical laser scanner data.

Differential geometry preliminaries and terminology. For a smooth oriented surface $\mathscr{S}$ let us denote by $k_{\max }$ and $k_{\min }$ the maximal and minimal principal curvatures, $k_{\max } \geq k_{\min }$. Let $\mathbf{t}_{\max }$ and $\mathbf{t}_{\text {min }}$ be the corresponding principal directions. Consider the derivatives of the principal curvatures along their corresponding curvatures directions $e_{\max }=\partial k_{\max } / \partial \mathbf{t}_{\max }$ and $e_{\min }=\partial k_{\min } / \partial \mathbf{t}_{\min }$. Notice that $e_{\max }$ and $e_{\min }$ are defined locally in neighborhoods of non-umbilical points. The extrema of the principal curvatures along their curvature directions are given by the zero-crossings of $e_{\max }$ and $e_{\min }$, and the ridges and valleys are characterized by

$$
\begin{aligned}
& e_{\max }=0, \quad \partial e_{\max } / \partial \mathbf{t}_{\max }<0, \quad k_{\max }>\left|k_{\min }\right|, \quad \text { (ridges), } \\
& e_{\min }=0, \quad \partial e_{\min } / \partial \mathbf{t}_{\min }>0, \quad k_{\min }<-\left|k_{\max }\right| \quad \text { (valleys). }
\end{aligned}
$$

Since the ridges and valleys turn into each other as surface orientation is changed, without loss of generality we can consider only the ridges. 


\section{Curvature Estimation}

We assume that a given mesh $\mathscr{M}$ is rescaled so that the length of a main diagonal of an axis-aligned bounding cube is equal to $d .^{1}$

Implicit surface fitting. For each mesh vertex $\mathbf{v}$ we estimate the normal vector $\mathbf{n}(\mathbf{v})$ as the normalized weighted sum of the normals of triangles incident to the vertex. Then we construct an implicit surface $F(\mathbf{x})=0$ approximating the set of the mesh vertices and normals.

The approach of [Ohtake et al. 2003b], which we use to build $F(\mathbf{x})=0$, can be considered an extension of techniques developed in [Floater and Iske 1996; Iske and Levesley 2002] from scattered height data fitting to scattered 3D data fitting. It consists of the following. Given a set of points $\mathscr{P}$ sampled from a smooth surface, a hierarchy of point sets $\left\{\mathscr{P}_{1}, \mathscr{P}_{2}, \ldots, \mathscr{P}_{n}=\mathscr{P}\right\}$ is created such that $\mathscr{P}_{k}$ is a simplification of $\mathscr{P}_{k+1}$ and is obtained from $\mathscr{P}_{k+1}$ by clustering subsets of $\mathscr{P}_{k+1}$. Then a bottom-up approach is employed to construct implicit surfaces $F_{k}(\mathbf{x})=0$ interpolating $\mathscr{P}_{k}$, $k=1, \ldots, n$, respectively. The interpolation process starts from $\mathscr{P}_{1}$. Given $F_{k}(\mathbf{x})=0$ interpolating $\mathscr{P}_{k}$, a partition of unity (PU) approximation of $\mathscr{P}_{k+1}$ is constructed by blending local quadratic approximations of $\mathscr{P}_{k+1}$. Function $F_{k+1}(\mathbf{x})$ whose zero-level set interpolates $\mathscr{P}_{k+1}$ is then obtained as the sum of $F_{k}(\mathbf{x})$, the PU approximation of $\mathscr{P}_{k+1}$, and a sum of CS-RBFs.

The modifications we propose to adapt the method of [Ohtake et al. 2003b] for our needs consist of employing smoother Wendland's $C^{3} \cap P D_{3}$ functions [Floater and Iske 1996] with double support size compared with that used in [Ohtake et al. 2003b], blending local linear approximations instead of quadratic ones, and switching from interpolation to approximation via a regularization of the corresponding RBF interpolation matrices: instead of inverting $\mathrm{RBF}$ interpolation matrix $\boldsymbol{\Phi}$, its regularization $\boldsymbol{\Phi}+\lambda \mathbf{I}$ is inverted. We use a regularization parameter $\lambda=0.1$ in all our experiments.

Estimating curvatures and their derivatives. After the implicit surface $F(\mathbf{x})=0$ approximating mesh $\mathscr{M}$ is constructed, for each mesh vertex $\mathbf{v}$, its projection $\hat{\mathbf{v}}$ onto the implicit surface is found and the curvature tensor and curvature derivatives at $\mathbf{v}$ are estimated by those computed analytically at $\hat{\mathbf{v}}$.

To find $\hat{\mathbf{v}}$ we use the Newton-iterative process:

$$
\hat{\mathbf{v}}_{0}=\mathbf{v}, \quad \hat{\mathbf{v}}_{k+1} \leftarrow \hat{\mathbf{v}}_{k}-\frac{F\left(\hat{\mathbf{v}}_{k}\right) \nabla F\left(\hat{\mathbf{v}}_{k}\right)}{\left\|\nabla F\left(\hat{\mathbf{v}}_{k}\right)\right\|^{2}} \text { until } \frac{\left|F\left(\hat{\mathbf{v}}_{n}\right)\right|}{\left\|\nabla F\left(\hat{\mathbf{v}}_{n}\right)\right\|}<\frac{\varepsilon}{d},
$$

where $\varepsilon$ is a user-specified precision parameter and $d$ is the length of a main diagonal of the bounding cube of $\mathscr{M}$. We have found that stopping criterion in (1) with $\varepsilon=10^{-4}$ is quite sufficient for an accurate projection of the mesh vertices onto the approximating implicit surface. Usually only a few iterations of (1) are required since $\mathbf{v}$ is already close to $F(\mathbf{x})=0$.

The unit normal at $\mathbf{v}$ is estimated by $\mathbf{n}=\left(n_{1}, n_{2}, n_{3}\right)=\nabla F /|\nabla F|$ at $\hat{\mathbf{v}}$. The two non-zero eigenvalues of $\nabla \mathbf{n}$ and their corresponding eigenvectors are used to approximate the curvature tensor at $\mathbf{v}$. The derivative of a principal curvature $k$ along its corresponding principal direction $\mathbf{t}=\left(t_{1}, t_{2}, t_{3}\right)$ is given by [Monga et al. 1992], [Porteous 1994, Exercise 11.8]:

$$
e=\nabla k \cdot \mathbf{t}=\left(F_{i j l} t^{i} t^{j} t^{l}+3 k F_{i j} t^{i} n^{j}\right) /|\nabla F|
$$

where $F_{i j}$ and $F_{i j l}$ denote the second and third partial derivatives of $F(\mathbf{x})$, respectively, and the Einstein summation convention is used. Now $e_{\max }$ and $e_{\min }$ at mesh vertex $\mathbf{v}$ are estimated according to (2) computed at $\hat{\mathbf{v}}$.

\section{Tracing Ridges}

Detection of ridge vertices. Once we can estimate the curvature tensor and curvature derivative $e_{\max }(\mathbf{v})$ at each mesh vertex $\mathbf{v}$,

${ }^{1} d=20 \sqrt{3}$ in our current implementation. we are ready to check whether the mesh edge $\left[\mathbf{v}_{1}, \mathbf{v}_{2}\right]$ contains a ridge vertex.

We flip $\mathbf{t}_{\max }\left(\mathbf{v}_{2}\right)$ if the angle between $\mathbf{t}_{\max }\left(\mathbf{v}_{1}\right)$ and $\mathbf{t}_{\max }\left(\mathbf{v}_{2}\right)$ is obtuse: $\mathbf{t}_{\max }\left(\mathbf{v}_{2}\right) \leftarrow-\mathbf{t}_{\max }\left(\mathbf{v}_{2}\right), e_{\max }\left(\mathbf{v}_{2}\right) \leftarrow-e_{\max }\left(\mathbf{v}_{2}\right)$. Next we check the following conditions:

$k_{\max }(\mathbf{v})>\left|k_{\min }(\mathbf{v})\right|$ for $\mathbf{v}=\mathbf{v}_{1}, \mathbf{v}_{2}$ and $e_{\max }\left(\mathbf{v}_{1}\right) e_{\max }\left(\mathbf{v}_{2}\right)<0$,

where the latter verifies whether curvature derivative $e_{\max }$ has a zero-crossing on $\left[\mathbf{v}_{1}, \mathbf{v}_{2}\right]$. Finally we apply a simple derivative test

$$
e_{\max }\left(\mathbf{v}_{i}\right)\left[\left(\mathbf{v}_{3-i}-\mathbf{v}_{i}\right) \cdot \mathbf{t}_{\max }\left(\mathbf{v}_{i}\right)\right]>0 \quad \text { with } i=1 \text { or } 2
$$

to determine whether $e_{\max }$ attains a maximum on $\left[\mathbf{v}_{1}, \mathbf{v}_{2}\right]$.

If (3) and (4) are satisfied, we use linear interpolation to approximate a zero-crossing of $e_{\max }$ on $\left[\mathbf{v}_{1} \mathbf{v}_{2}\right]$

$$
\mathbf{p}=\frac{\left|e_{\max }\left(\mathbf{v}_{2}\right)\right| \mathbf{v}_{1}+\left|e_{\max }\left(\mathbf{v}_{1}\right)\right| \mathbf{v}_{2}}{\left|e_{\max }\left(\mathbf{v}_{1}\right)\right|+\left|e_{\max }\left(\mathbf{v}_{2}\right)\right|}
$$

and consider $\mathbf{p}$ a ridge vertex

The above procedure would not work properly near the umbilical points $\left(k_{\max }=k_{\min }\right)$ since the principal directions $\mathbf{t}_{\max }$ and $\mathbf{t}_{\min }$ are not defined at the umbilics and a practical detection of $\mathbf{t}_{\max }$ and $\mathbf{t}_{\min }$ near the umbilics is unstable. Fortunately it can be easily shown [Belyaev et al. 1997, Theorem 18.2.8] that the loci of maxima of $k_{\max }$ and of minima of $k_{\min }$ along their corresponding curvature lines do not pass through the generic (typical) umbilics. Therefore the ridge-valley lines do not approach the generic umbilical points.

Another potential danger arises from points where the angle between mesh edge $\left[\mathbf{v}_{3-i}, \mathbf{v}_{i}\right]$ and principal direction $\mathbf{t}_{\max }\left(\mathbf{v}_{i}\right), i=1,2$, is very close to zero or even vanishes, since the derivative test (4) may fail there. However, in our numerical experiments, we haven't observed any side effect related to such failures.

Connecting ridge vertices. If two ridge vertices are detected on edges of a mesh triangle, they are connected by a straight segment. If all three edges of a mesh triangle contain ridge vertices, the vertices are connected with the centroid of the triangle formed by the vertices.

Thresholding. We measure the strength of a ridge line by the integral of $k_{\max }$ along the line and use the trapezoid approximation of the integral

$$
\int k_{\max } d s \approx \sum_{i} \frac{k_{\max }\left(\mathbf{p}_{i}\right)+k_{\max }\left(\mathbf{p}_{i+1}\right)}{2}\left\|\mathbf{p}_{i}-\mathbf{p}_{i+1}\right\|=T .
$$

Note that $T$ is a scale-independent threshold. We use linear interpolation to estimate $k_{\max }$ at the vertices of the polyline approximation of the ridge line:

$$
k_{\max }(\mathbf{p})=\frac{\left|e_{\max }\left(\mathbf{v}_{2}\right)\right| k_{\max }\left(\mathbf{v}_{1}\right)+\left|e_{\max }\left(\mathbf{v}_{1}\right)\right| k_{\max }\left(\mathbf{v}_{2}\right)}{\left|e_{\max }\left(\mathbf{v}_{1}\right)\right|+\left|e_{\max }\left(\mathbf{v}_{2}\right)\right|},
$$

where $\left[\mathbf{v}_{1}, \mathbf{v}_{2}\right]$ is the mesh edge containing $\mathbf{p}$.

We ignore those ridge lines for which the right hand-side of (5) is less than a user-specified value of threshold $T$.

Thickness adjustment. For visualization purposes, we determine the thickness of each segment $\left[\mathbf{v}_{1}, \mathbf{v}_{2}\right]$ of a ridge line according to the principal curvature values $k_{\max }\left(\mathbf{v}_{1}\right)$ and $k_{\max }\left(\mathbf{v}_{2}\right)$. We have found that visually pleasant results are obtained if the thickness is proportional to the arithmetic mean of $k_{\max }\left(\mathbf{v}_{1}\right)$ and $k_{\max }\left(\mathbf{v}_{2}\right)$ with the same proportionality coefficient for all ridge segments.

We use trilinear interpolation for estimating the principal curvature at a triple junction of a ridge line. Then all the three ridge line segments incident to the junction are assigned their own thicknesses.

\section{Results and Discussion}

The ridge-valley lines detected on complex shapes with many small wrinkles usually have poor connectivity. For example, small wrinkles on a salient surface crease spawn to many small disconnected ridges and valleys crossing the crease. Various techniques used to 
reduce fragmentation of line features detected on a mesh include mesh smoothing, geodesic snakes [Grenader and Miller 1998], and hysteresis thresholding [Hubeli and Gross 2001; DeCarlo et al. 2003]. The approach we use for reducing fragmentation of the ridge-valley lines can be considered a variation of mesh smoothing. To estimate the curvature tensor and curvature derivative $e_{\max }$ and $e_{\min }$ at mesh vertices $\mathscr{P}$, we use implicit approximation $F_{k}(\mathbf{x})=0$ of $\mathscr{P}_{k}, k<n$, a simplified version of $\mathscr{P}$ (described in Section 2).

Figure 1 compares the ridge-valley patterns detected on an eye part of the $1 \mathrm{~mm}$ Michelangelo's David head model when implicit approximations of simplified sets $\mathscr{P}_{7}$ (middle image) and $\mathscr{P}_{8}$ (right image) are used. ${ }^{2}$ The fragmentation of the ridges and valleys is substantially reduced when simpler implicit function approxima-

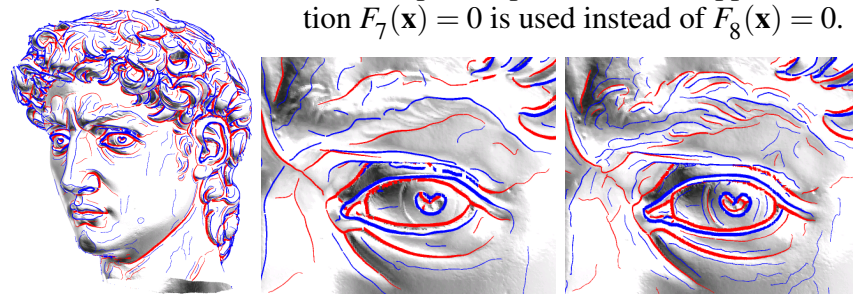

Figure 1: Blue ridges and red valleys detected on Michelangelo's David head model approximated by $F_{7}(\mathbf{x})=0$ (left and middle) and $F_{8}(\mathbf{x})=0$ (right), $\mathrm{T}=0.5$.

Figure 2 shows patterns of ridges and valleys detected on various triangle meshes. Notice how well the ridge-valley lines capture both salient shape features and small shape variations.

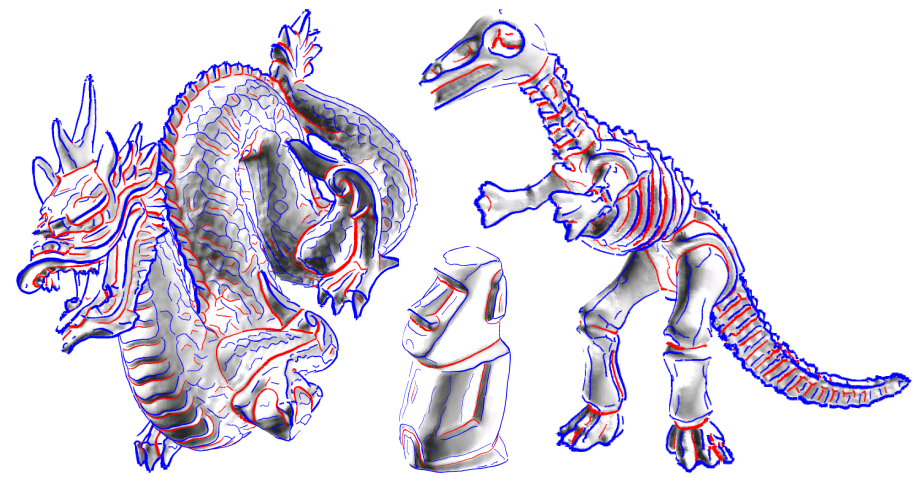

Figure 2: Ridges and valleys detected on various models, $T=0.5$.

Further, the images of Figure 2 suggest that the ridge-valley lines convey perceptually important information, and we also foresee applications of our approach in simulating artistic drawings of $3 \mathrm{D}$ objects. The usefulness of the ridge-valley structures for nonphotorealistic rendering is not surprising [Ma and Interrante 1997; DeCarlo et al. 2003]. Figure 3 shows the ridges and valleys detected on the Stanford Buddha model for two different values of parameter $T$. One can notice that salient ridges and valleys ( $T$ is large) are particularly good for highlighting garment wrinkles.

Figure 4 demonstrates how much geometric information is carried by salient ridges and valleys. Thus, similar to multiscale image edges [Mallat and Zhong 1992], multiscale ridge-valley lines can be used for shape coding purposes.

According to our numerical experiments, detecting the ridgevalley lines from the principal curvature tensor via non-maximum suppression and hysteresis thresholding, as suggested in [Hubeli and Gross 2001; DeCarlo et al. 2003], often produces hairy patterns of poorly connected ridges and valleys. This is especially true for dense irregular meshes approximating surfaces with smooth curvature variations. On the other hand, discriminating between curvature maxima and minima via the second derivative test $\nabla e \cdot \mathbf{t} \lessgtr 0$,

\footnotetext{
${ }^{2}$ The full resolution is achieved at octree depth 10 .
}
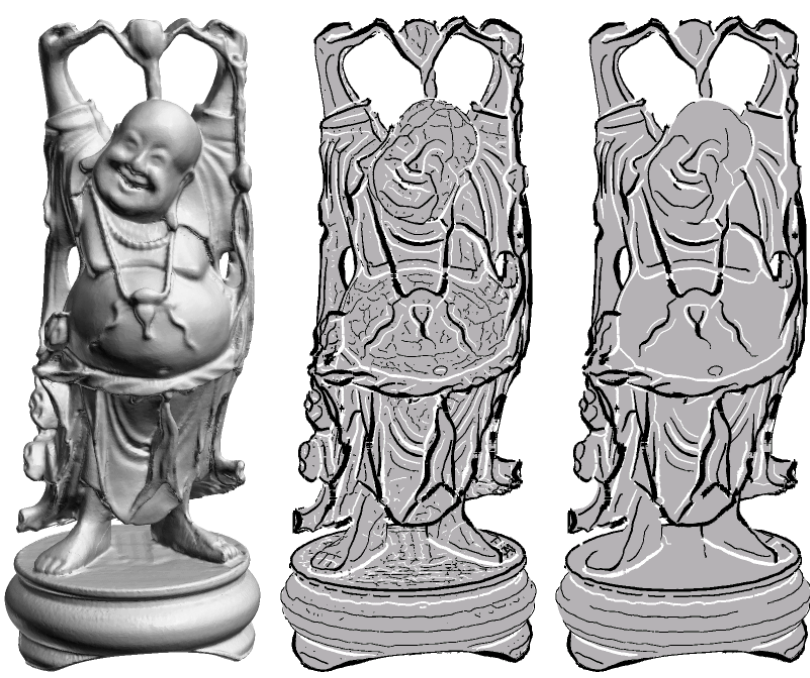

Figure 3: Filtering ridges (black) and valleys (white) according to their strength controlled by parameter $T$. Middle: $T=0$. Right: $T=2$.
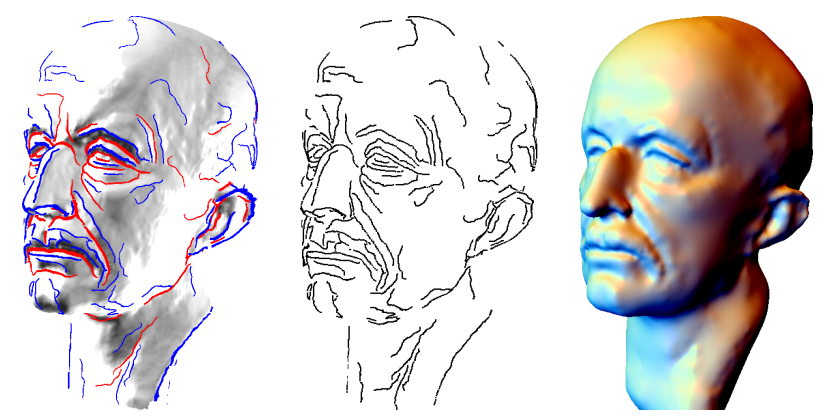

Figure 4: Left: salient ridges and valleys detected on the Max-Planck bust model, $T=2$. Middle: the ridges and valleys alone are sufficient for recognizing the model. Right: only the ridge-valley vertices and mesh normals at them are used for shape reconstruction via implicit surface fitting.

where $\nabla e \cdot \mathbf{t}$ is computed analytically is not practical for processing models with complex geometry.

In Figure 5, four different methods for detecting the ridge-valley lines on implicit surfaces are compared. The first method (the leftmost image) is the most accurate: $\nabla e_{\max } \cdot \mathbf{t}_{\max }$ and $\nabla e_{\min } \cdot \mathbf{t}_{\text {min }}$ are computed analytically. The second one (second from the left image) is the method of this paper: we polygonize the surface (a marching cubes algorithm is used) and then follow the procedure of Section 2. One can notice how accurate our ridge-valley detection procedure is. The third method (rightmost) consists of using a modified MPU fitting method ${ }^{3}$ instead of the hierarchical CS-RBFs of Section 2. The modified MPU fitting method has a local nature and does not deliver a sufficiently accurate surface approximation. Finally, the fourth method (the rightmost image) consists of computing the curvature tensor at the vertices of the marching cubes mesh analytically and then applying the non-maximum suppression and hysteresis thresholding operations mentioned above. The resulting ridges and valleys are fragmented. Much worse results are obtained if the curvature tensor is estimated from the mesh.

The two left images of Figure 5 also demonstrate that ridgevalley lines capture small surface details which are below human visual sensitivity and suggests their suitability for quality evaluation of various surface fitting methods.

Our implicit surface fitting procedure (described in Section 2) uses a simple octree-based hierarchical clustering which is orientation dependent. That dependence is reduced when local shape

\footnotetext{
${ }^{3}$ In order to achieve $C^{3}$ continuity we use a smoother blending function to compare with that used in the original MPU method [Ohtake et al. 2003a].
} 

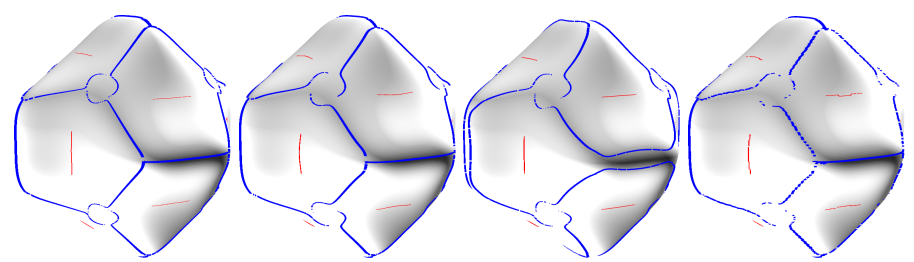

Figure 5: Ridges (blue) and valleys (red) detected on implicit dodecahedronlike surface $x^{6}+y^{6}+z^{6}+20\left(x^{4} y^{2}+y^{4} z^{2}+z^{4} x^{2}\right)=1$. Left: second-order curvature derivatives computed analytically are used for discriminating between curvature maxima and minima. Middle left: the method described in this paper is employed. Middle right: modified MPU fitting is used. Right: the curvature tensor is computed analytically and then non-maximum suppression and hysteresis thresholding are applied.

approximations are built. Nevertheless, since the curvature extrema are very delicate surface features, small differences in the ridgevalley patterns detected on the same model under different orientation conditions are observed. Figure 6 demonstrates orientation insensitivity of geometrically salient ridge-valley lines.
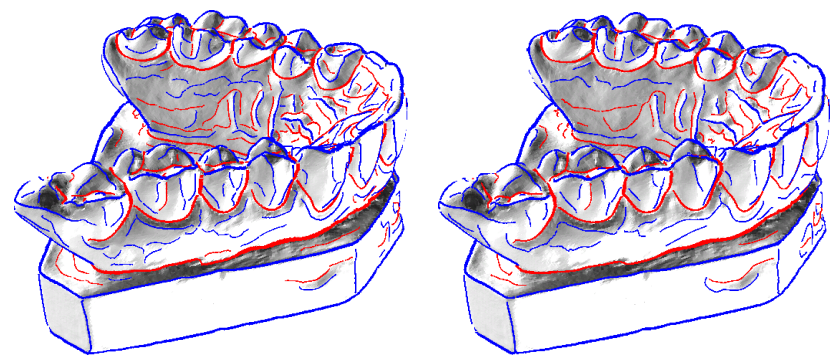

Figure 6: Ridge- valley patterns detected on Teeth Casting model (left) and on the same model after two $\pi / 4$ rotations about two orthogonal axes are applied (right), $T=0.5$.

Another limitation of our approach is its low speed. For example, detecting the ridge-valley lines on the $1 \mathrm{~mm}$ Michelangelo's David head model consisting of more than $4 \mathrm{M}$ triangles takes about an hour on a low-end PC. The most time-consuming stage is computing the curvature tensor and curvature derivatives $e_{\max }$ and $e_{\min }$ at mesh vertex projections $\hat{\mathbf{v}}$, since the approximating implicit surface $F(\mathbf{x})=0$ is globally defined.

Motivated by the results shown in Figure 4 and the similarity between the ridge-valley lines and image edges, we plan to adapt the edge-based image compression method [Mallat and Zhong 1992] for shape compression purposes.

Acknowledgments. The models are courtesy of the Stanford Computer Graphics Lab, Cyberware, and MPI für Informatik.

\section{References}

Belyaev, A. G., Anoshrina, E. V., And Kunit, T. L. 1997. Ridges, ravines, and singularities. In A. T. Fomenko, and T. L. Kunii, Topological Modeling for Visualization, Springer, 375-383. Chapter 18.

Carr, J. C., Beatson, R. K., Cherrie, J. B., Mitchell, T. J., Fright, W. R., McCallum, B. C., AND Evans, T. R. 2001. Reconstruction and representation of $3 \mathrm{D}$ objects with radial basis functions. In Proc. ACM SIGGRAPH 2001, 67-76.

Cazals, F., And Pouget, M. 2003. Estimating differential quantities using polynomial fitting of osculating jets. In Symposium on Geometry Processing, 177-187.

Cohen-Steiner, D., And Morvan, J.-M. 2003. Restricted Delaunay triangulations and normal cycle. In Proc. 19th ACM Symp. on Comput. Geom., 312-321.

DeCarlo, D., Finkelstein, A., Rusinkiewicz, S., and Santella, A. 2003. Suggestive contours for conveying shape. ACM Trans. on Graphics 22, 3, 848-855. Proc. ACM SIGGRAPH 2003.
FloAter, M. S., AND IsKe, A. 1996. Multistep scattered data interpolation using compactly supported radial basis functions. J. Comput. Applied Math. 73, 65-78.

GoldFeather, J., AND InTERRAnTe, V. 2004. A novel cubic-order algorithm for approximating principal direction vectors. ACM Trans. on Graphics 23, 1, $45-63$.

GRenader, U., AND Miller, M. I. 1998. Computational anatomy: An emerging discipline. Quarterly of Applied Mathematics 56, 4, 617-694.

Gumhold, S., Wang, X., AND MCLeod, R. 2001. Feature extraction from point clouds. In Proc. 10th International Meshing Roundtable, 293-305.

Hallinan, P. L., Gordon, G. G., Yuille, A. L., Giblin, P., And Mumford, D. 1999. Two- and Tree-Dimensional Patterns of the Face. A K Peters.

HosaKa, M. 1992. Modeling of Curves and Surfaces in CAD/CAM. Springer, Berlin.

Hubeli, A., AND Gross, M. 2001. Multiresolution feature extraction from unstructured meshes. In Proc. IEEE Visualization 2001, 287-294.

ISKe, A., AND LeVeSLEY, J. 2002. Multilevel scattered data approximation by adaptive domain decomposition. Tech. Rep. TUM-M0208, Technische Universität München.

Kent, J. T., Mardia, K. V., And West, J. 1996. Ridge curves and shape analysis. In The British Machine Vision Conference 1996, 43-52.

Koenderink, J. J. 1990. Solid Shape. MIT Press.

LitTle, J. J., AND ShI, P. 2001. Structural lines, TINs and DEMs. Algorithmica 30, 2, 243-263.

López, A. M., F. Lumbreras, F., AND Serrat, J. 1998. Creaseness from level set extrinsic curvature. In Proc. ECCV'98, Springer, 156-169.

MA, K.-L., AND INTERRANTE, V. 1997. Extracting feature lines from 3D unstructured grids. In Proc. IEEE Visualization 1997, 285-292.

Mallat, S., AND Zhong, S. 1992. Characterization of signals from multiscale edges. IEEE Trans. Pattern Anal. Mach. Intell. 4, 7, 710-732.

Meyer, M., Desbrun, M., Schröder, P., ANd Barr, A. H. 2003. Discrete differential-geometry operators for triangulated 2-manifolds. In Visualization and Mathematics III, Springer, H.-C. Hege and K. Polthier, Eds., 35-58.

Monga, O., Benayoun, S., And Faugeras, O. 1992. From partial derivatives of 3-D density images to ridge lines. In Proc. CVPR'92, IEEE, 354-359.

Ohtake, Y., Belyaev, A., Alexa, M., Turk, G., And Seidel, H.-P. 2003. Multi-level partition of unity implicits. ACM Trans. on Graphics 22, 3, 463-470. Proc. ACM SIGGRAPH 2003.

Ohtake, Y., Belyaev, A. G., And Seidel, H.-P. 2003. A multi-scale approach to 3D scattered data interpolation with compactly supported basis functions. In Shape Modeling International 2003, 153-161.

Page, D. L., Sun, Y., Koschan, A., Paik, J., AND Abidi, M. 2002. Normal vector voting: Crease detection and curvature estimation on large, noisy meshes. Journal of Graphical Models 64, 1-31.

Pauly, M., Keiser, R., And Gross, M. 2003. Multi-scale feature extraction on point-sampled models. Computer Graphics Forum 22, 3, 281-289. Eurographics 2003 issue.

Pennec, X., Ayache, N., And Thirion, J. P. 2000. Landmark-based registration using features identified through differential geometry. In Handbook of Medical Imaging, I. N. Bankman, Ed. Academic Press, 499-513.

PORTEOUS, I. R. 1994. Geometric Differentiation for the Intelligence of Curves and Surfaces. Cambridge University Press, Cambridge.

Stylianou, G., and Farin, G. 2003. Crest lines extraction from 3D triangulated meshes. In Hierarchical and Geometrical Methods in Scientific Visualization, Springer, G. Farin, B. Hamann, and H. Hagen, Eds., 269-281

Watanabe, K., And Belyaev, A. G. 2001. Detection of salient curvature features on polygonal surfaces. Computer Graphics Forum 20, 3 , 385-392. Eurographics 2001 issue. 